\title{
Transverse momentum spectra of fermions and bosons produced in strong abelian fields
}

\author{
Vladimir V. Skokov \\ Bogoliubov Laboratory of Theoretical Physics, \\ Joint Institute for Nuclear Research, \\ 141980, Dubna, Russia \\ Péter Lévai \\ RMKI Research Institute for Particle and Nuclear Physics, \\ P.O. Box 49, Budapest 1525, Hungary
}

(Dated: 25 October 2004)

\begin{abstract}
We study the transverse momentum spectra of fermions and bosons produced in strong, timedependent abelian field. The transverse size of the abelian field is finite, similarly to color strings and ropes. Different time-dependent field strengths are investigated in a kinetic model, and transverse momentum spectra are calculated for fermions and bosons. These spectra display exponential or polynomial behavior at high $p_{T}$, depending on the given time dependence. We compare our spectra to lattice result for a classical gluon field and obtain surprisingly good agreement in certain cases.
\end{abstract}

PACS numbers: 24.85.+p,25.75.-q, 12.38.Mh

\section{INTRODUCTION}

Ultrarelativistic heavy ion collisions at SPS and RHIC energies $(\sqrt{s}=10-200 \mathrm{AGeV})$ provided an enormous volume of experimental data on particle production [1]. The microscopic mechanisms of hadron production are not fully understood and many different descriptions coexist to explain these data. Transverse momentum spectra of produced hadrons have been measured in a wide momentum region (e.g. $0 \leq p_{T} \leq 15 \mathrm{GeV}$ at RHIC), and can become a decisive test between different perturbative and non-perturbative models of hadron formation.

Investigations of $p p$ collisions at high energies led to the introduction of chromoelectric flux tube ('string') models, where these tubes are connecting the quark and diquark constituents of colliding protons [2, 3, 4]. Strings are good examples of how to convert the kinetic energy of a collision into field energy. When these flux tubes become unstable, new hadrons will be produced via quark-antiquark and diquark-antidiquark pair production. Such models can describe experimental data successfully at low $p_{T}, p_{T}<2-3 \mathrm{GeV}$. At higher $p_{T}$ perturbative QCD-based models are working [5, 6, 7].

In heavy ion reactions finite number of nucleons collide and the number of produced strings scales with the number of participant nucleons. Since hadron production at low $p_{T}$ scales with participant nucleons in a wide energy range, string models could reproduce available data surprisingly well in the soft region at SPS energies [2, 3, 4]. However, the obtained high density for strings strengthened the idea of string melting and the formation of color ropes [8], which fill the finite transverse extension partially or completely. Following these ideas, measured strangeness enhancement was explained successfully by rope formation [4]. This result has indicated the possible formation of extended color fields at SPS energies.

At RHIC and LHC energies the string density is ex- pected to be so large that a strong gluon field will be formed in the whole available transverse volume. Furthermore, the gluon number will be so high that a classical gluon field as the expectation value of the quantum field can be considered and investigated in the reaction volume. The properties of such non-abelian classical fields and details of gluon production were studied very intensively during the last years, especially asymptotic solutions 9, 10]. Fermion production was calculated recently [11, 12]. Lattice calculations were performed also to describe strong classical fields under finite space-time conditions 13, 14].

Fermion pair production together with boson pair production were investigated by kinetic models of particle production from strong abelian 15, 16, 17, 18, 19, 20, 21, 22 and non-abelian 23] fields. These calculations concentrated mostly on bulk properties, the time dependence of energy and particle number densities.

Our main interest is the transverse momentum distribution of produced fermions and bosons. Before performing non-abelian kinetic model calculation, we would like to understand the role of time dependence, the interplay between production and annihilation rates in a kinetic approach and the influence of finite transverse size on the transverse momentum distributions.

In this paper we summarize our results applying a kinetic model with a time-dependent abelian external field characterized by finite transverse geometry. We concentrate on transverse momentum spectra for produced particles. Section 2 summarizes the field theoretical backgrounds for boson and fermion production in strong abelian field. The kinetic equation is explained briefly in Section 3. In Section 4 the influence of time dependence on fermionic and bosonic transverse momentum spectra is displayed and the obtained results are compared to lattice calculations. In Section 5 we discuss our results. 


\section{BOSONS AND FERMIONS IN EXTERNAL FIELD}

Let us consider a massive boson field $\phi$ in an external classical abelian vector field, $A^{\mu}$. The Lagrangian

$$
\mathcal{L}_{\phi}=D_{\mu}^{*} \phi^{*} D^{\mu} \phi-m_{+}^{2} \phi^{*} \phi
$$

leads to the equation of motion

$$
\left(D^{\mu} D_{\mu}+m_{+}^{2}\right) \phi=0,
$$

where $D_{\mu}=\partial_{\mu}+i e_{+} A_{\mu}$ with bosonic charge $e_{+}$. The bosonic mass is labelled by $m_{+}$.

We will choose a longitudinally dominant vector field in Hamilton gauge with the 4-potential $A^{\mu}=\left(0,0,0, A^{3}\right)$, which is the most appropriate for our investigation in the ultrarelativistic energy region.

To imitate confinement properties of Yang-Mills fields, the component $A^{3}$ is limited in the transverse direction, and a finite 'flux tube' radius $r_{0}$ is considered. The external field is cylindrically symmetric. It vanishes outside the tube, $A^{3}\left(t, r>r_{0}, \varphi, x_{3}\right)=0$, and it is homogeneous inside the flux tube, $A^{3}\left(t, r \leq r_{0}, \varphi, x_{3}\right) \equiv$ $A^{3}(t)$ [17, 18, 19].

The Klein-Gordon equation (2) reads for the boson field $\phi\left(t, r, \varphi, x_{3}\right)$ as

$$
\left[\partial_{0}^{2}-\nabla_{\perp}^{2}-\partial_{3}^{2}-2 i e_{+} A_{3}(t) \partial_{3}+e_{+}^{2} A_{3}^{2}(t)+m_{+}^{2}\right] \phi=0,
$$

where the transverse Laplace operator is given by

$$
\nabla_{\perp}^{2}=\frac{\partial^{2}}{\partial r^{2}}+\frac{1}{r} \frac{\partial}{\partial r}+\frac{1}{r^{2}} \frac{\partial^{2}}{\partial \varphi^{2}} .
$$

For the bosonic field function in eq. (3) we are looking for the following solution:

$$
\phi\left(t, r, \varphi, x_{3}\right)=N e^{i l \varphi} e^{-i k_{3} x_{3}} T(t) R(r) .
$$

One equation is obtained for the time-dependent part,

$$
\ddot{T}(t)+\left[m_{+}^{2}+\left(k_{3}-e_{+} A_{3}(t)\right)^{2}+\epsilon^{2}\right] T(t)=0,
$$

and one for the spatial dependence,

$$
r^{2} R^{\prime \prime}(r)+r R^{\prime}(r)+\left(\epsilon^{2} r^{2}-l^{2}\right) R(r)=0 .
$$

Here $\epsilon$ is the separation constant which will be fixed later.

Considering flux-tube boundary condition for $A_{3}(t)$ and the constraint $R\left(\epsilon r_{0}\right)=0$ on the surface of the flux tube, the solution for the boson field is

$$
\phi_{n l, k_{3}}\left(t, r, \varphi, x_{3}\right)=e^{-i k_{3} x_{3}} \frac{T_{n l, k_{3}}(t) J_{l}\left(\epsilon_{n l} r\right)}{\sqrt{\pi} r_{0} J_{l}^{\prime}\left(\epsilon_{n l} r_{0}\right)} e^{i l \varphi},
$$

where $\epsilon_{n l} r_{0}$ is the $n^{t h}$ zero of the Bessel function $J_{l}$ and the constant $J_{l}^{\prime}\left(\epsilon_{n l} r_{0}\right)$ appears during the normalization of the field function.
The energy of the bosonic quasiparticles reads

$$
\omega_{+}^{2}\left(t, k_{T}, k_{3}\right)=m_{+}^{2}+\epsilon_{n l}^{2}+P_{+}^{2},
$$

where $P_{+}=k_{3}-e_{+} A_{3}(t)$ is the kinetic longitudinal momenta and $\epsilon_{n l}$ labels the discrete transverse momenta. In Section 3 the transverse momenta spectra of the newly produced bosons will be determined at these discrete transverse momentum values, $\epsilon_{n l}$.

Massive fermions $\left(m_{-}, e_{-}\right)$can be described similarly in the presence of the above external classical abelian field $A^{\mu}(t)$. Considering the fermionic Lagrangian

$$
\mathcal{L}_{\psi}=\bar{\psi}\left(i \gamma^{\mu} D_{\mu}-m_{-}\right) \psi
$$

one obtains the corresponding equation of motion

$$
\left(i \gamma^{\mu} \partial_{\mu}-e_{-} \gamma^{3} A_{3}(t)-m_{-}\right) \psi(x)=0 .
$$

The solution of eq. (11) is wanted in the form of

$$
\psi(x)=\left(i \gamma^{\mu} \partial_{\mu}-e_{-} \gamma^{3} A_{3}(t)+m_{-}\right) \widehat{\psi}(x),
$$

where an auxiliary field $\widehat{\psi}(x)$ was introduced [24]. In the cylindrically symmetric case, one obtains the following equation for $\widehat{\psi}\left(t, r, \phi, x_{3}\right)$ :

$$
\begin{array}{r}
{\left[\partial_{0}^{2}-\nabla_{\perp}^{2}-\partial_{3}^{2}-2 i e_{-} A_{3}(t) \partial_{3}+e_{-}^{2} A_{3}^{2}(t)\right.} \\
\left.+i e_{-} \gamma^{0} \gamma^{3} \partial_{0} A_{3}+m_{-}^{2}\right] \widehat{\psi}=0 .
\end{array}
$$

Now we can look for the solution of eq.113) in a form similar to eq. (5):

$$
\widehat{\psi}\left(t, r, \varphi, x_{3}\right)=N e^{i l \varphi} e^{-i k_{3} x_{3}} \widehat{T}(t) \widehat{R}(r) \chi_{\lambda} .
$$

Eigenvector of matrix $\gamma_{0} \gamma_{3}$ is labelled by $\chi_{\lambda}, \lambda= \pm 1$. During the solution of eq. (13), the radial equation for $\widehat{R}(r)$ leads to eq.(7). Thus, one obtains $\widehat{R}(r) \equiv R(r)$ and the same radial solutions with the same separation constants $\epsilon_{n l}$ as above. The one-particle energy for the auxiliary field is the following:

$$
\widehat{\omega}_{-}^{2}\left(t, k_{T}, k_{3}\right)=m_{-}^{2}+\epsilon_{n l}^{2}+P_{-}^{2} .
$$

Here $P_{-}(t)=k_{3}-e_{-} A_{3}(t)$ labels longitudinal momenta. Time-dependent component $\widehat{T}(t)$ satisfies the equation

$$
\ddot{\widehat{T}}(t)+\left[\widehat{\omega}_{-}^{2}\left(t, k_{T}, k_{3}\right)+i e_{-} \dot{A}_{3}(t)\right] \widehat{T}(t)=0 .
$$

Using eq. (12) for the solution of the auxiliary field, one can reconstruct the fermion field $\psi\left(t, r, \varphi, x_{3}\right)$. The dispersion relation in eq. (15) remains valid for these fermions.

The finite radius of the flux tube determines a minimal transverse energy, $\epsilon_{10}=0.2404 / r_{0}$. For $p p$ collisions we obtain $\epsilon_{10}\left(r_{0}=1 \mathrm{fm}\right)=47.4 \mathrm{MeV}$, for central $A u A u$ collisions it is $\epsilon_{10}\left(r_{0}=6.5 \mathrm{fm}\right)=7.3 \mathrm{MeV}$, which are very small, but nonzero values. 


\section{THE KINETIC EQUATION}

The kinetic equation with time-dependent external field can be constructed on the basis of the calculated bosonic and fermionic field functions. Using a timedependent Bogoliubov transformation approach (see e.g. Refs. [24, 25]) or a quasiparticle representation approach 21], one obtains the following kinetic equation:

$$
\frac{\partial f_{ \pm}\left(t, k_{3}\right)}{\partial t}+e_{ \pm} E(t) \frac{\partial f_{ \pm}\left(t, k_{3}\right)}{\partial k_{3}}=\mathcal{S}_{ \pm}\left(t, k_{3}\right) \text {. }
$$

Here $E(t)=-\dot{A}_{3}(t)$. One-particle distribution functions for bosons $(+)$ and fermions $(-)$ are labelled by $f_{ \pm}\left(t, k_{3}\right)$. We note that the measurable longitudinal momentum of the produced particle has the value of $P_{ \pm}$.

Equation (17) will be solved at the discrete transverse momenta $\epsilon_{n l}$, which depend on the transverse radius $\left(r_{0}\right)$ of the chromoelectric field. Indeed, eq. (17) can be solved at continuous transverse momenta and the solutions at corresponding $\epsilon_{n l}$ will be selected.

The source term $\mathcal{S}_{ \pm}\left(t, k_{3}\right)$ contains information about the time evolution of the system and is determined as

$$
\begin{gathered}
\mathcal{S}_{ \pm}\left(t, k_{3}\right)=\frac{1}{2} W_{ \pm}\left(t, k_{3}\right) \int_{-\infty}^{t} d t^{\prime} \bar{W}_{ \pm}\left(t, t^{\prime} ; k_{3}\right) \\
\left\{\left[1 \pm 2 f_{ \pm}\left(t^{\prime}, k_{3}\right)\right] \cos \left[2 \int_{t^{\prime}}^{t} d \tau \bar{\omega}_{ \pm}\left(t, \tau ; k_{3}\right)\right]\right\}
\end{gathered}
$$

where

$$
\begin{aligned}
\bar{\omega}_{ \pm}^{2}\left(t, t^{\prime} ; k_{3}\right) & =m_{ \pm}^{2}+\epsilon_{n l}^{2}+\bar{P}_{ \pm}^{2}\left(t, t^{\prime}\right) \\
\bar{P}_{ \pm}\left(t, t^{\prime}\right) & =k_{3}+e_{ \pm} \int_{t^{\prime}}^{t} d \tau E(\tau) \\
\bar{W}_{ \pm}\left(t, t^{\prime} ; k_{3}\right) & =\frac{e_{ \pm} E(t) \bar{P}_{ \pm}\left(t, t^{\prime}\right)}{\bar{\omega}_{ \pm}^{2}\left(t, t^{\prime} ; k_{3}\right)}\left[\frac{\sqrt{m_{ \pm}^{2}+\epsilon_{n l}^{2}}}{\bar{P}_{ \pm}\left(t, t^{\prime}\right)}\right]^{2 s_{ \pm}}
\end{aligned}
$$

Overlined notation indicates appropriate asymptotic behavior, namely $W_{ \pm}\left(t, k_{3}\right) \equiv \bar{W}_{ \pm}\left(t, t^{\prime} \rightarrow-\infty ; k_{3}\right)$. Similar asymptotics are valid for $P_{ \pm}(t)$ and $\omega_{ \pm}(t)$.

The difference in boson and fermion production appears through the spin factor in eq. (21), namely $s_{+}=0$ for bosons and $s_{-}=1 / 2$ for fermions.

Now we introduce new auxiliary functions, $v_{ \pm}$and $u_{ \pm}$, and rewrite the integro-differential eq.(17) in a more convenient form containing full derivatives:

$$
\begin{aligned}
& \dot{f}_{ \pm}=\frac{1}{2} W_{ \pm} v_{ \pm}, \\
& \dot{v}_{ \pm}=W_{ \pm}\left(1 \pm 2 f_{ \pm}\right)-2 \omega_{ \pm} u_{ \pm}, \\
& \dot{u}_{ \pm}=2 \omega_{ \pm} v_{ \pm} .
\end{aligned}
$$

This system of differential equations is solved for all sets of $\left\{\epsilon_{n l}, k_{3}\right\}$, which correspond to the different transverse and longitudinal momenta.
The functions $f_{ \pm}, v_{ \pm}, u_{ \pm}$start with zero initial value, describing a system without any bosons or fermions at the beginning. Assuming different time dependences for the external field, $E(t)$, we obtain different particle productions. We would like to note that produced charged particles generate an inner field which interferes with the external one [21]. This effect is neglected here, as well as a possible collision term, $\mathcal{C}_{ \pm}\left(t, k_{3}\right)$, on the right-hand side of eq. (17) 26]. A given external field can mimic a certain self-consistent solution as expansion, collision or back reaction influenced time dependence of the field.

\section{NUMERICAL RESULTS}

In heavy ion collisions, one can assume three different types of time dependence for the chromoelectric field to be formed: a) pulse-like field develops with a fast increase, which is followed by a fast fall in the field strength; b) formation of a constant field $\left(E_{0}\right)$ is maintained after the fast increase in the initial time period; c) scaled decrease of the field strength appears, which is caused by particle production and/or transverse expansion, and the decrease is elongated in time much further than the pulse-like assumption.

Figure 11displays three sets for the time dependence of the external field to investigate the above three different physical scenarios numerically:

$$
\begin{aligned}
E_{\text {pulse }}(t) & =E_{0} \cdot[1-\tanh (t / \delta)] \\
E_{\text {const }}(t) & = \begin{cases}E_{\text {pulse }}(t) & \text { at } t<0 \\
E_{0} & \text { at } t \geq 0\end{cases} \\
E_{\text {scaled }}(t) & = \begin{cases}E_{\text {pulse }}(t) & \text { at } t<0 \\
\frac{E_{0}}{\left(1+t / t_{0}\right)^{\kappa}} & \text { at } t \geq 0\end{cases}
\end{aligned}
$$

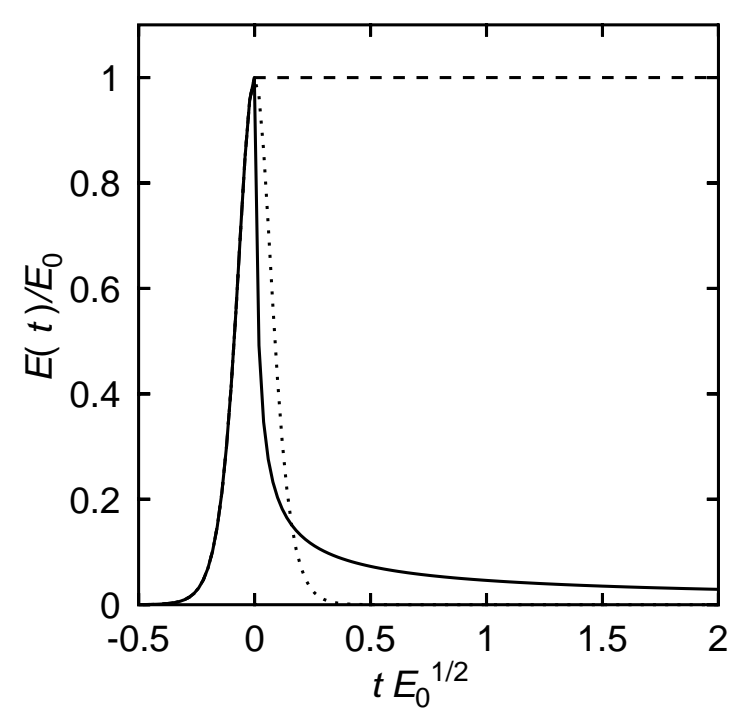

FIG. 1: The time dependence of external field $E(t)$ in three physical scenarios: a) pulse (dotted line); b) constant field, $E_{0}$ (dashed line); c) scaled decrease (solid line). 


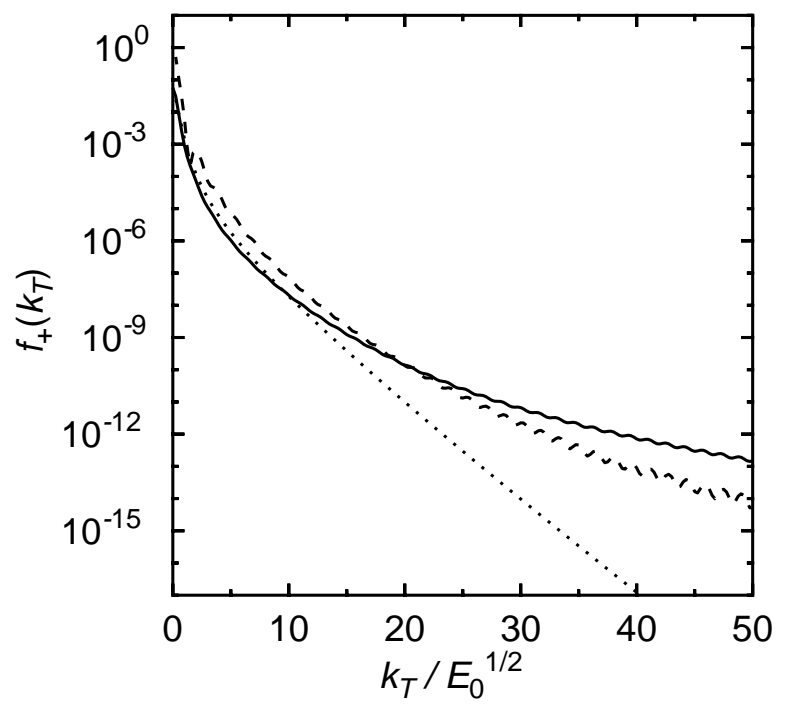

FIG. 2: The transverse momentum spectra for bosons in the three physical scenarios (see Fig. 1 and text for explanation).

Here we choose $\delta=0.1 / E_{0}^{1 / 2}$, which corresponds to RHIC energies, and $\kappa=2 / 3$ to indicate a longitudinally scaled Bjorken expansion with $t_{0}=0.01 / E_{0}^{1 / 2}$. In fact, the whole time dependence is scaled by $E_{0}{ }^{1 / 2}$.

Figures 2 and 3 display the obtained transverse momentum spectra in midrapidity at time $t=2 / E_{0}^{1 / 2}$ for bosons and fermions. Pulse-type time dependence leads to exponential spectra (dotted lines), $f_{ \pm} \propto \exp \left(k_{T} / T_{ \pm}\right)$, where the slope value is $T_{+}=1.6 \cdot E_{0}^{1 / 2}$ for bosons and $T_{-}=1.45 \cdot E_{0}^{1 / 2}$ for fermions. In the other two cases, we obtain non-exponential spectra, which are generated by the long-lived electric field. The transverse spectra from constant (dashed lines) and scaled (solid lines) fields are

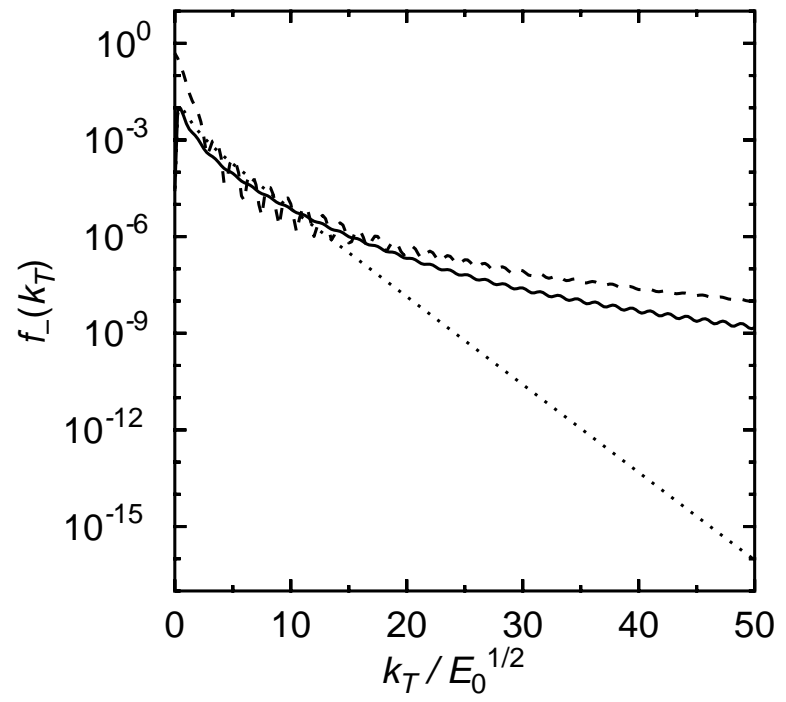

FIG. 3: The transverse momentum spectra for fermions in the three physical scenarios (see Fig. 1 and text for explanation).

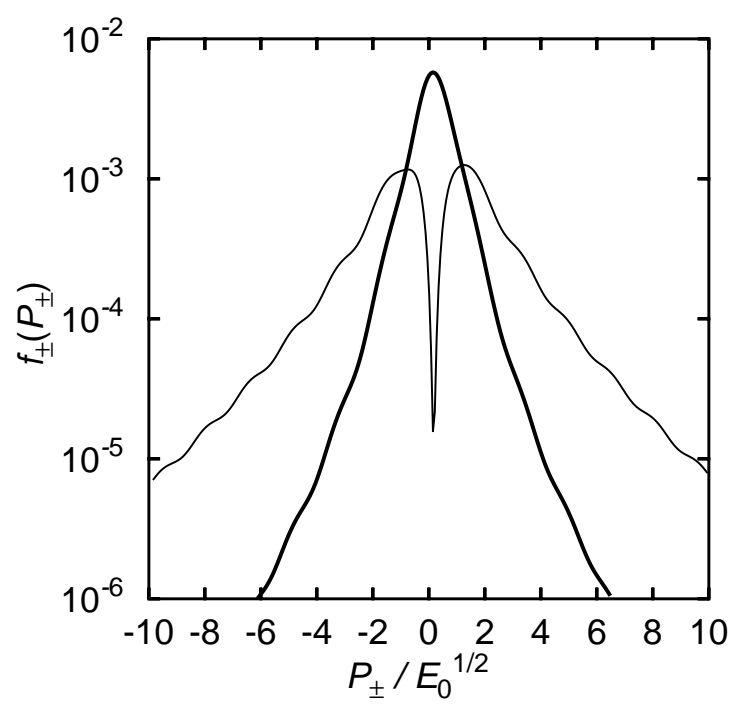

FIG. 4: Longitudinal momentum spectra for fermions (thick line) and bosons (thin line) at $k_{T} / E_{0}^{1 / 2}=1$ and $t=2 / E_{0}^{1 / 2}$.

close, because the production and annihilation rates balance each other. Slight differences appear because of the fast fall of the scaled field immediately after $t=0$. We note here, that indeed we have discrete transverse momenta, $\epsilon_{n l}$, but with such small steps, which allow us to display continuous transverse momentum spectra.

Comparing the boson and fermion production in midrapidity, one can recognize the large difference in the production rates and see a clear fermion dominance at all transverse momenta. The reason of this dominance can be understood from Fig. 4, which displays the longitudinal particle spectra, $f_{ \pm}\left(P_{ \pm}\right)$, at fixed transverse momenta, $k_{T} / E_{0}^{1 / 2}=1$, and time, $t=2 / E_{0}^{1 / 2}$. Fermion spectra (thick line) have a reasonable value around zero longitudinal momenta (which means midrapidity), but boson spectra (thin line) display a 'dip'-like structure here. The presence of this 'dip' is related to the structure of the transition rate $\bar{W}\left(t, t^{\prime} ; k_{3}\right)$ in eq. (21) and the longitudinal dominance of the electric field defined via $A_{3}(t)$ : at zero longitudinal momenta $\left(P_{ \pm}\right)$there is no boson production, but fermion production is characterized by a well defined finite value.

At extremely high-energy heavy ion collisions we expect this longitudinal dominance of the chromoelectric field. If transverse electric field components become comparable to the longitudinal one at lower energies, then the boson yield will be much larger. The presence of scattering term, $\mathcal{C}_{ \pm}$, can increase the yield in midrapidity from the neighbouring rapidity cells, where the yield is large, even if a longitudinal field component, $A^{3}$, exists alone.

In Refs. 13 transverse momentum spectra for gluons were determined in SU(3) classical field theory. Comparison between this non-abelian and our abelian calculation can be made, if we fix both field strength at the same value. The energy density of the classical gluon field is given in Refs. [13] as $\varepsilon_{S U 3}=0.17 \Lambda_{s}^{4} / g^{2}$, where 


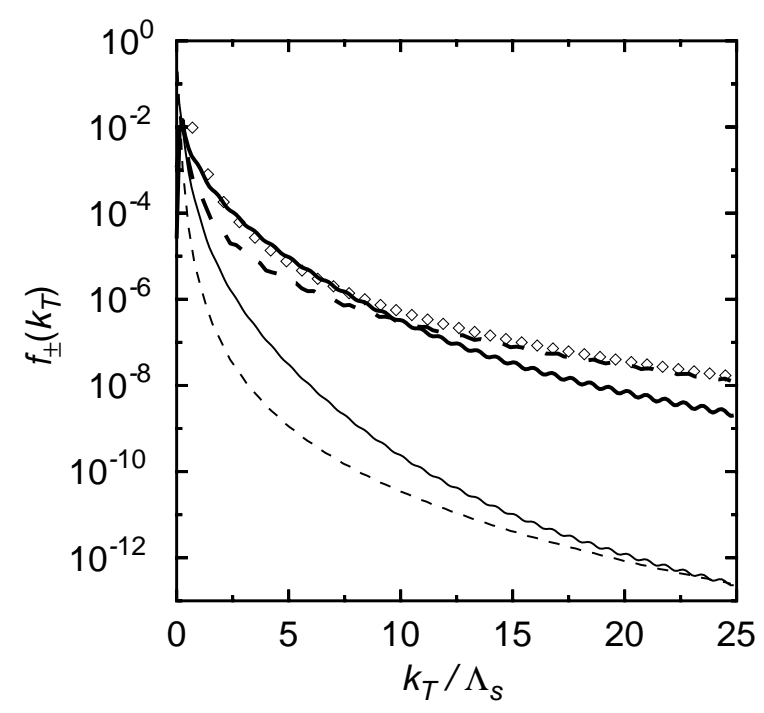

FIG. 5: Transverse momentum spectra for fermions (thick lines) and bosons (thin lines) from our calculation with scaled time evolution, $E_{\text {scaled }}(t)$, and gluon production (diamonds) from $\mathrm{SU}(3)$ lattice-QCD calculation [13].

$g=2\left(\alpha_{s}=0.33\right)$. In our calculation, the initial energy density is related to the field strength $E_{0}(t=0)$ : $\varepsilon=E_{0}^{2} / 2$. Assuming the same 'initial' energy density in the abelian and the non-abelian case, we obtain $E_{0}^{1 / 2} / \Lambda_{s}=0.54$. Now we can rescale our transverse momentum spectra from Figures 2 and 3 - we choose the results with scaled time-dependent field, $E_{\text {scaled }}$ (solid lines). In Figure 5 our results at RHIC energies for fermion (thick solid line) and boson (thin solid line) are displayed together with non-abelian result for classical gluons [13] (open diamonds). Our fermion spectra and the lattice result on classical gluons overlap in the mo- mentum region $2<k_{T} / \Lambda_{s}<10$. Possible parametrization of the two spectra are very close to each other and display the $\log \left(k_{T} / \Lambda_{s}\right) \cdot\left(k_{T} / \Lambda_{s}\right)^{-4}$ behavior known from Ref. [13] and from perturbative QCD [27]. An acceptable slight modification of the time evolution in eq. (27) could lead to a full overlap, even at high- $k_{T}$. This can be demonstrated at $k_{T} / \Lambda_{s}>15$ with $\delta=0.01 / E_{0}^{1 / 2}$ for fermion production (thick dashed line).

\section{DISCUSSION}

In this paper we investigated fermion and boson production from a strong abelian field in a kinetic model. Assuming ultrarelativistic limit and a longitudinally dominant external field, boson production remains relatively weak in the mid-rapidity region. However, fermion production is not effected by this setup and a fermion dominance appears in our kinetic model. Introducing different parametrizations for the time evolution of the external field, we obtain different transverse momentum spectra for fermions and bosons. Only time evolution determines if these spectra are exponential or polynomial. Investigating fermion transverse momentum spectra, our results with abelian external field overlap with the spectra obtained in lattice-QCD calculations for classical gluons at the same energy density.

\section{Acknowledgments}

We thank V. Toneev and G. Fai for stimulating discussions. This work was supported in part by Hungarian grant OTKA-T043455, MTA-JINR Grant, and RFBR grant No. 03-02-16877.
[1] Quark Matter'04 Conference Proceedings (Ed. by H.G. Ritter and X.N. Wang), J. Phys. G 30 (2004) S633.

[2] B. Andersson et al., Phys. Rep. 97 (1983) 31; Nucl. Phys. B281 (1987) 289; Z. Phys. C57 (1993) 485.

[3] X.N. Wang, M. Gyulassy, Phys. Rev. D44 (1991) 3501; Comput. Phys. Commun. 83 (1994) 307.

[4] H. Sorge, Phys. Rev. C52 (1995) 3291.

[5] R.D. Field, Application of Perturbative QCD, AddisonWesley, 1989.

[6] X.N. Wang, Phys. Rev. C61 (2000) 064910.

[7] Y. Zhang et al., Phys. Rev. C65 (2002) 034903.

[8] T.S. Biró et al., Nucl. Phys. B245 (1984) 449.

[9] L.D. McLerran, Lecture Notes Phys. 583 (2002) 291; hep-ph/0402137 and references therein.

[10] E. Iancu and R. Venugopalan, hep-ph/0303204 and references therein.

[11] F. Gelis, R. Venugopalan, Phys. Rev. D69 (2004) 014019.

[12] J.P. Blaizot, F. Gelis, R. Venugopalan, Nucl. Phys. A743 (2004) 57.

[13] A. Krasnitz, Y. Nara, and R. Venugopalan, Phys. Rev. Lett. 87 (2001) 192302.
[14] T. Lappi, Phys. Rev. C67 (2003) 054903.

[15] G. Gatoff, et al. Phys. Rev. D36 (1987) 114.

[16] Y. Kluger et al., Phys. Rev. Lett. 67 (1991) 2427.

[17] G. Gatoff and C.Y. Wong, Phys. Rev. D46 (1992) 997.

[18] C.Y. Wong, et al. Phys. Rev. D51 (1995) 3940.

[19] J.M. Eisenberg, Phys. Rev. D51 (1995) 1938.

[20] D.V. Vinnik, et al., Few-Body Syst. 32 (2002) 23.

[21] V.N. Pervushin, et al. accepted in Int. Mod. Phys. A. (hep-th/0307200).

[22] D.D. Dietrich, Phys. Rev. D68 (2003) 105005.

[23] A.V. Prozorkevich, S.A. Smolyansky, and S.V. Ilyin, (hep-ph/0301169).

[24] A.A. Grib, S.G. Mamaev, and V.M. Mostepanenko, Vacuum Quantum effects in strong external fields, (Friedmann Laboratory Publishing, St. Petersburg, 1994).

[25] S.A. Smolyansky et al., (hep-ph/9712377).

[26] V.V. Skokov, Proc. of 16th Int. Baldin Conference, Dubna, 2002, (hep-ph/0210099).

[27] M. Gyulassy, L. McLerran, Phys. Rev. C56 (1997) 2219. 